\title{
Simulating Electric Double Layer Capacitance of Mesoporous Electrodes with Cylindrical Pores
}

\author{
Julian Varghese, Hainan Wang, and Laurent Pilon ${ }^{\mathrm{z}}$ \\ University of California, Los Angeles, Henry Samueli School of Engineering and Applied Science Mechanical \\ and Aerospace Engineering Department, Los Angeles, California 90095, USA
}

\begin{abstract}
This paper aims to numerically assess the effects of electrolyte properties and electrode morphology on the capacitance of electric double layer capacitors (EDLCs) made of mesoporous electrodes consisting of ordered cylindrical pores in non-aqueous electrolytes. Simulations solved a three-dimensional modified Poisson-Boltzmann model. They accounted for the finite size of ions and field-dependent electrolyte permittivity while the pores were perpendicular to the current collector. The effects of pore radius, porosity, effective ion diameter, and electrolyte field-dependent permittivity on the diffuse layer gravimetric capacitance were investigated systematically in order to determine key parameters affecting EDLCs' performance. The simulations showed that reducing the ion effective diameter and the pore radius resulted in the strongest increase in diffuse layer gravimetric capacitance up to a critical radius below which the capacitance reaches a plateau. Increasing the electrode porosity also increased the diffuse layer gravimetric capacitance. Accounting for more realistic field-dependent permittivity was found to significantly reduce the predicted diffuse layer gravimetric capacitance. Finally, accounting for the contribution of the Stern layer to the total capacitance was essential in predicting experimental data for a wide range of porous activated carbon electrodes and non-aqueous electrolytes. (C) 2011 The Electrochemical Society. [DOI: 10.1149/1.3622342] All rights reserved.
\end{abstract}

Manuscript submitted March 4, 2011; revised manuscript received July 13, 2011. Published August 8, 2011.

Supercapacitors or electric double layer capacitors (EDLCs) are energy storage devices that store electric charge in the electric double layer forming at an electrode-electrolyte interface. ${ }^{1-7}$ Typical commercial EDLCs use carbon electrodes featuring large surface area $^{1-4}$ and resulting in devices with large gravimetric and volumetric capacitances. This gives EDLCs very large energy density compared to conventional capacitors but not as large as that of rechargeable batteries. ${ }^{1-4}$ The operational voltage of a single electric double layer capacitor is limited by the breakdown potential of the electrolyte, which is about $1 \mathrm{~V}$ for aqueous electrolytes and less than $3 \mathrm{~V}$ for organic electrolytes. ${ }^{2-7}$ EDLCs store charge physically and therefore do not experience any major change in material microstructure and composition during charging/discharging. By contrast, batteries store energy chemically which involves changes in the electrode material structure between its charged and discharged states. $^{2-7}$ Therefore, unlike batteries, EDLCs have an essentially unlimited charge/discharge cycle life. In addition, EDLCs can be charged and discharged within a few seconds compared with minutes or hours for batteries. ${ }^{2-7}$ Thus, EDLCs have larger power density than batteries. ${ }^{2-7}$ These properties make EDLCs a very attractive option as energy storage devices in a variety of applications ranging from wireless communications to regenerative braking systems in hybrid electric vehicles. ${ }^{2-7}$ However, the energy density of EDLCs needs to increase to make them competitive with batteries.

An accurate and rigorous numerical tool would be very useful in systematically identifying (i) the physical phenomena controlling the behavior of EDLCs and (ii) the design parameters affecting their performance. It should account for the most significant physical phenomena. It should also be validated against experimental measurements. It can then serve as a powerful tool to guide the experimental fabrication of nanostructured electrodes with the desired performance. This paper presents numerical simulations of charge storage in electric double layer capacitors. The goal is to identify the main physical phenomena responsible for EDLC performance including the effects of electrode morphology and electrolyte properties. It is then used to predict experimental data reported in the literature for EDLCs with a wide range of electrode morphologies and non-aqueous electrolytes.

\section{Background}

Analytical models._Gouy-Chapman model.-The classical theory of electrokinetics dates back to the late 19 th century. ${ }^{8}$ Helm-

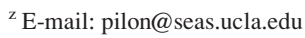

holtz ${ }^{8}$ proposed a double layer model consisting of a simple separation of charges at an electrode/electrolyte interface similar to a conventional parallel-plate capacitor. One plate is the actual electrode separated from an imaginary plate by an electrolyte layer called the Helmholtz layer of thickness $d_{H} \cdot{ }^{9}$ The Helmholtz model predicts the specific area capacitance (in $\mathrm{F} / \mathrm{m}^{2}$ ) of the system to be $C_{H}=\varepsilon_{r} \varepsilon_{0} / d_{H}$ where $\varepsilon_{r}$ is the dielectric constant of the electrolyte and $\varepsilon_{0}$ is the permittivity of free space. Here, $C_{H}$ is independent of surface potential and electrolyte concentration. ${ }^{9}$ However, this was not observed experimentally. ${ }^{10}$

The Helmholtz model was modified by Gouy ${ }^{11}$ and Chapman ${ }^{12}$ with the consideration that the ion concentration should be continuous in the electrolyte solution. In fact, ions are driven by electrostatic forces coupled with random thermal agitation. ${ }^{13}$ This results in the so-called diffuse layer. The Gouy-Chapman model includes the effects of both the electrode potential and the bulk ionic concentration on the local ionic concentration and potential field in the electrolyte. It uses the Boltzmann distribution function to describe the local ion concentration field within the electrolyte. ${ }^{8}$ Chapman $^{12}$ derived and solved the steady-state Poisson-Boltzmann equation to predict electric potential in the diffuse layer. However, even for very dilute solutions, this theory predicts unrealistically large ion concentrations for surface potentials of just a fraction of $1 \mathrm{~V}$. This can be attributed to the fact that the ions are assumed to be point charges while, in reality, they have a finite size. ${ }^{13}$ Moreover, in practice the surface potential in EDLCs is in the order of a few volts depending on the electrolyte solution. Therefore, this theory cannot be used to model actual EDLCs with a typical surface potential of 1 $\mathrm{V}$ and bulk electrolyte concentration of $1 \mathrm{~mol} / \mathrm{l}$.

Stern model.-Stern ${ }^{14}$ proposed an electric double layer model accounting for the size and the specific (covalent) adsorption of ions. The author combined the Helmholtz and Gouy-Chapman models to explicitly describe the ion concentration in two distinct regions namely: (1) the inner region near the electrode called the Stern layer and (2) the outer region called the diffuse layer. ${ }^{14}$ Grahame $^{15}$ improved Stern's model by considering that in the Stern layer, adsorption of anions and cations at the electrode surface lead to different double layer thicknesses. ${ }^{10}$ The model developed by Stern ${ }^{14}$ and Grahame ${ }^{15}$ is usually referred to as the Stern model. ${ }^{16}$ The Stern layer consists of ions adsorbed by specific (also called covalent) forces and non-specific (or electrostatic) forces. The inner Helmholtz plane (IHP) and outer Helmholtz plane $(\mathrm{OHP})^{10}$ are used to distinguish the locations of these two types of adsorbed ions. It should be noted that there is no electroneutrality within the electric double layer. ${ }^{13}$ 
Effects of finite ion size (steric effects).- The dilute-solution theories assume point size ions in a mean-field or continuum approximation. ${ }^{17}$ However, they break down when the ion concentration increases and effects such as steric repulsion and many-body electrostatic correlation become significant. ${ }^{18}$ Many attempts have been made in the literature to account for the steric effects in the electrolyte solution. ${ }^{17-19}$ Among the various Modified Poisson-Boltzmann (MPB) theories, the continuum models based on the local-density and mean-field approximations are arguably the most convenient both mathematically and numerically. ${ }^{18}$ The reader is referred to the review by Bazant et al. ${ }^{18}$ for a more in-depth description of the different MPB theories. Here, a particular MPB theory based on the Bikerman-Freise formula ${ }^{18}$ was used to model EDLCs. This MPB model is one of the oldest and simplest MPB models as well as analytically tractable under certain assumptions.

Electrode materials.-Most commercial EDLCs are made of carbon electrodes including activated carbon, ${ }^{20-22}$ carbon nanotubes, ${ }^{22-25}$ templated carbons, ${ }^{1,26}$ and carbon aerogels. ${ }^{27,28}$ Carbon materials are attractive for electrodes due to their versatility, easy processability, non-toxicity, high chemical stability, low density, good electrical conductivity, high surface area, and relatively low cost. $^{2,3,7}$ The design of electrodes using carbon materials requires ${ }^{2,3,7}$ (i) high specific surface area to ensure high capacitance, (ii) appropriate pore-size and pore distribution to permit easy access of ions in the electrolyte to the electrode surface, and (iii) small electrical resistance. In addition, nanostructured porous materials provide great potential as electrode materials for achieving EDLCs with large energy density. Indeed, these materials have much larger surface area per unit volume than traditional macroporous materials. Furthermore, advances in nanotechnologies have made the synthesis of mesoporous materials possible with controlled morphology using repeatable and inexpensive synthesis routes. ${ }^{29-35}$ However, the optimum morphological parameters resulting in maximum EDLC performance remain to be identified.

Electrolyte.-The performance of electric double layer capacitors also strongly depends on the electrolyte. The operating potential of EDLCs is determined by the electrochemical stability window (or potential) of the electrolyte. ${ }^{5,10}$ Above that potential, electrolysis of the electrolyte occurs resulting in system failure and destruction. ${ }^{10}$ Both the energy and power densities of EDLCs increase with increasing operating voltage. Thus, electrolytes with high electrochemical operational window for electrolytes are desirable. ${ }^{5,10}$

Two frequently used aqueous electrolytes are $\mathrm{KOH}$ and $\mathrm{H}_{2} \mathrm{SO}_{4}$. Both of them have high electrical conductivity resulting in lower device impedance and faster response time. ${ }^{5}$ However, the maximum operating potential of aqueous electrolyte solutions is typically restricted to $1 \mathrm{~V}$ due to water's electrochemical stability window of $1.23 \mathrm{~V}^{4-6}$

Moreover, higher voltage in EDLCs can be achieved by using organic electrolytes which can typically operate at electrode potential larger than $2 \mathrm{~V}^{4,6}$ Organic electrolytes used in commercial EDLCs generally consist of $1 \mathrm{M}$ triethylmethylammonium tetrafluoroborate $\left(\mathrm{TEMA}-\mathrm{BF}_{4}\right)$ in acetonitrile $(\mathrm{AN})$ or propylene carbonate $(\mathrm{PC}){ }^{5}$ However, major issues remain associated with the use of organic electrolytes including their (i) high cost, (ii) low electrical conductivity compared with aqueous electrolytes, (iii) low dielectric constant leading to smaller capacitance, as well as (iv) safety concerns due to the flammability and toxicity of their organic solvents. ${ }^{4}$

Simulations of EDLCs.-The models discussed previously have been used extensively for simulating EDLCs despite their inherent limitations. Huang et al. ${ }^{36-38}$ used the Helmholtz model to predict the specific area capacitance of EDLCs based on single spherical and cylindrical electrode carbon particles as well as single cylindrical pore. They predicted that the capacitance per unit surface area (or specific area capacitance) of spherical and cylindrical electrode particles decreases with increasing electrode diameter larger than 2 $\mathrm{nm}$. However, it increases with pore diameter for electrodes with cylindrical pores. Their model also predicted the "anomalous" increase of specific area capacitance for pore diameter less than 2 $\mathrm{nm}$ as observed experimentally in Ref. 39 . However, the electrolyte permittivity was used as an empirical parameter to match the theoretical specific area capacitance with experimental data. ${ }^{36-38}$

Henstridge et al. $^{40}$ predicted the capacitance of EDLCs with electrodes made of carbon nanotubes by simulating one cylindrical nanoelectrode particle. The authors solved the Gouy-Chapman model numerically in one-dimensional radial coordinate system. The electrode radius ranging from 0.32 to $50 \mathrm{~nm}$, the surface electric potential $\psi_{s}$ was less than $0.25 \mathrm{~V}$, while the aqueous electrolyte concentration $c_{\infty}$ was less than $0.01 \mathrm{~mol} / \mathrm{l}$. Their model predicted significant increase in double layer capacitance for electrode radius less than $20 \mathrm{~nm}$. However, this model cannot be used for actual EDLCs where the typical electrolyte concentration is about $1 \mathrm{~mol} / \mathrm{l}$ and steric effects become significant (Effects of finite ion size section).

Huang et al. ${ }^{41}$ used the Stern model to investigate the effects of the shape and geometry of a single nanopore on the specific area capacitance of EDLCs. They explored two-dimensional "slit" and cylindrical pores with width and diameter ranging from 2 to $16 \mathrm{~nm}$. The dielectric permittivity $\varepsilon_{r}=9.73$ was imposed empirically in the Stern layer based on the value previously fitted in Refs. 36 and 37. The electrolyte concentration was $c_{\infty}=1.0 \mathrm{~mol} / \mathrm{l}$ and the electrode surface potential was $\psi_{s}=1 \mathrm{~V}$. However, the Gouy-Chapman-Stern model breaks down for such concentration and potential since ions can no longer be treated as point-charges as demonstrated in Ref. 42.

The above mentioned simulations of EDLCs suffered from several limitations. First, the Gouy-Chapman and Stern models do not account for the finite ion size and thus are not valid for actual EDLCs with concentration larger than $1 \mathrm{~mol} / \mathrm{l}$. Second, the above simulations only focused on one- or two-dimensional electrode geometry. Thus, these studies were not adequate to facilitate the design of three-dimensional mesoporous electrodes to enhance the energy density of EDLCs. Third, the electrolyte dielectric permittivity was assumed to be constant and sometimes was treated as a fitting parameter. However, the relative permittivity $\varepsilon_{r}$ of polar electrolytes is known to significantly decrease as the electric field increases due to the high orientation of individual electrolyte molecules. 43,44

Recently, Wang et al. ${ }^{45}$ solved the MPB model combined with Booth model to predict the specific area capacitance of closelypacked monodispersed sphere arrays with SC, BCC, and FCC packing morphologies having respective porosity of 52,68 , and $74 \%$ and various sphere diameters. The electrolyte solution was $\mathrm{TEA}_{-} \mathrm{BF}_{4}$ in $\mathrm{PC}$ at $1 \mathrm{~mol} / \mathrm{l}$ with effective ion diameter of $0.68 \mathrm{~nm}$. The numerical results established that the diffuse layer specific area capacitance of the sphere arrays significantly decreased when the field-dependent electrolyte permittivity was accounted for ${ }^{45}$. In addition, the authors also demonstrated that the SC packing features the largest diffuse layer specific area capacitance due to its largest electrolyte volume fraction while FCC had the lowest. Moreover, the predicted total specific area capacitance was in good agreement with the experimental data for EDLC with FCC arrangement of monodisperse mesoporous spheres $40 \mathrm{~nm}$ in diameter and non-aqueous TEA-BF 4 electrolyte solution reported in Ref. 46.

The goal of the present study is to systematically investigate the effect of electrode morphology and electrolyte properties on the capacitance of EDLCs. Here, EDLCs with three-dimensional electrodes consisting of ordered cylindrical pores were simulated. Parameters of particular interest included the pore radius, the electrode porosity, the electrolyte effective ion diameter and relative permittivity. Finally, numerical predictions for the total gravimetric capacitance were compared with experimental data reported in the literature. The present study differs from our previous one ${ }^{45}$ in the electrode morphology and the range of porosity, effective ion diameter, and dielectric constant parameters explored along with the use of experimental data for a wide range of electrode morphologies and different electrolytes. ${ }^{21,47,48}$ 


\section{Analysis}

Simulated configurations.- The idealized electrode morphology chosen for this study consisted of mesoporous films with highly-ordered cylindrical pores. Such electrodes can be synthesized using a templating process with appropriate precursors. ${ }^{49}$ Figure 1a shows the schematic of the simulated EDLC electrode consisting of identical cylindrical pores with axes perpendicular to the planar current collectors. The cylindrical pores of identical radius $r$ were arranged in a simple cubic lattice. The axes of adjacent pores were separated by a distance $2 f r$ where $f$ is a geometric parameter while the corresponding electrode porosity is given by $\phi=\pi / 4 f^{2}$.

Since the porosity of the experimental samples was not specified, the porosity was set to be constant and the radius of the cylindrical pores was varied from 0.5 to $10 \mathrm{~nm}$. Here, the porosity was specified to be $\phi=0.55$ for all simulations, unless otherwise mentioned, by maintaining the geometric parameter $f=1.2$. This value of porosity was chosen because it ensured that, for the range of pore radius considered, the simulated nanostructures had specific surface area ranging from 100 to $2,300 \mathrm{~m}^{2} / \mathrm{g}$ similar to that of experimental data reported in the literature. ${ }^{21,47}$ Note that mesoporous and microporous carbon electrodes with porosity $\phi$ ranging from 0.5 to 0.8 have been reported. $^{50}$

All cylindrical pores ran through the entire electrode thickness taken as $t=30 \mathrm{~nm}$. The thickness of the electrolyte region separating anode and cathode was assumed to be $140 \mathrm{~nm}$. Further increasing these values by a factor of two was found to have no effect on the predicted specific area capacitance (in $\mu \mathrm{F} / \mathrm{cm}^{2}$ ). Only the regions of the device occupied by the electrolyte solution was considered in the computational domain. In other words, the electrode and current collector were considered only so far as defining the electrode/electrolyte interface. Exploiting the anti-symmetry of the potential also allowed modeling of only half of the full unit cell. Here, we chose to model the anode. Additional symmetries within one half of the domain made it possible to analyze only one-eighth of the full unit cell as illustrated in Fig. 1b.

Assumptions.-To make the problem mathematically tractable, the following assumptions were made:

(1) The electrode material was a perfect conductor. Thus, the potential at the anode surface was uniform and equal to $+\psi_{s}$. (2) The anions and cations had the same effective diameter denoted by $a$. (3) The anions had a valency of $-z$ and the cations had a valency of $+z$. Indeed, the electrolytes considered (AN and PC) were binary and symmetric. (4) The temperature $T$ was uniform and equal to 298 $\mathrm{K}$ throughout the domain. (5) Advection of the electrolyte was

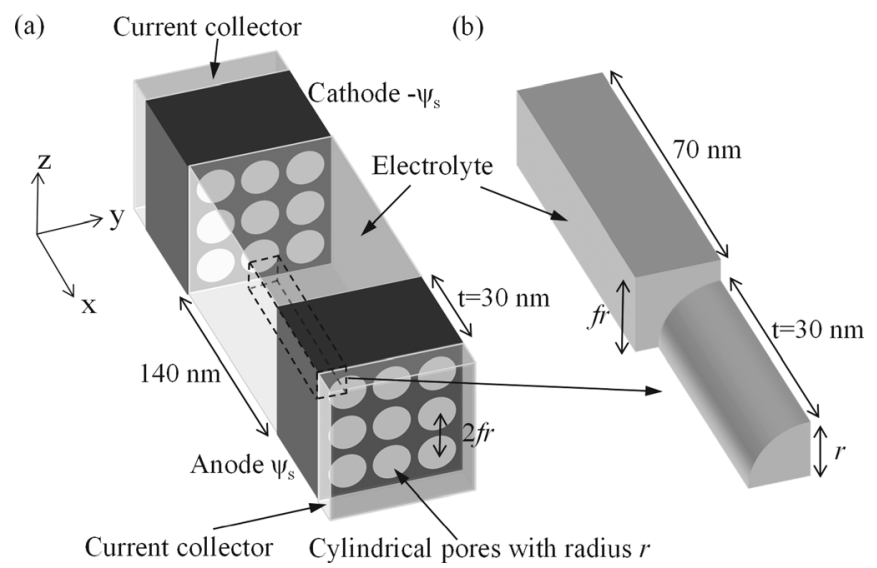

Figure 1. (a) Schematic of EDLC configuration with cylindrical pores of radius $r$ arranged in simple cubic lattice and (b) computational domain simulated. Electrode porosity is given by $\phi=\pi / 4 f^{2}$. assumed to be negligible. (6) The permittivity of the electrolyte was isotropic. (7) The continuum theory was assumed to be valid for all cases. Its validity has been examined in the literature ${ }^{51-54}$ and is typically accepted when the pore diameter is larger than $3-5 \mathrm{~nm} .{ }^{51-54}$

Governing equations.-The electrostatic potential, $\psi$ in the electrolyte solution satisfies the Poisson equation given by ${ }^{13}$

$$
\nabla \cdot\left(\varepsilon_{0} \varepsilon_{r} \nabla \psi\right)=-\sum_{i=1}^{N} z_{i} e N_{A} c_{i}
$$

where $z_{i}$ and $c_{i}$ are the valency and local molar concentrations of the ionic species $i, N_{A}=6.022 \times 10^{23} \mathrm{~mol}^{-1}$ is the Avogadro constant, $e=1.602 \times 10^{-19} \mathrm{C}$ is the unit charge, $N$ is the total number of ionic species. For a symmetric binary electrolyte, $N=2, z_{1}=-z_{2}=z$ and the local molar concentrations of the anions and cations, respectively denoted by $c_{1}$ and $c_{2}$, are given by ${ }^{18}$

$$
c_{i}=\frac{c_{\infty} \exp \left(-z e \psi / k_{B} T\right)}{1+2 v \sinh ^{2}\left(\frac{z e \psi}{2 k_{B} T}\right)} \quad(i=1 \text { or } 2)
$$

where $c_{\infty}$ is the ion's bulk molar concentration (in mol/l) at neutral state when $\psi=0$ while $k_{B}=1.3806 \times 10^{-23} \mathrm{~J} / \mathrm{K}$ is the Boltzmann constant. The packing parameter is defined as $v=2 a^{3} N_{A} c_{\infty}$ where $a$ is the effective diameter of solvated ions. ${ }^{19}$ It represents the ratio of the total bulk ion concentration to the maximum ion concentration $c_{\max }=1 /\left(N_{A} a^{3}\right)$, assuming a simple cubic ion packing. ${ }^{19}$ Therefore, $v$ should not be larger than unity for the model to be physically acceptable. ${ }^{18}$ As a result, the ion concentration given by Eq. 2 should not exceed the maximum concentration $c_{\text {max }} .{ }^{5}$

Combining Eqs. 1 and 2 yields the modified Poisson-Boltzmann (MPB) model expressed as ${ }^{17}$

$$
\nabla \cdot\left(\varepsilon_{0} \varepsilon_{r} \nabla \psi\right)=z e N_{A} c_{\infty} \frac{2 \sinh \left(\frac{z e \psi}{k_{B} T}\right)}{1+2 v \sinh ^{2}\left(\frac{z e \psi}{2 k_{B} T}\right)}
$$

From here on, the term MPB will refer to Eq. 3. Note that, in the limiting case of point charge ions $(a=v=0)$, the modified Boltzmann distribution (Eq. 2) reduces to the original Boltzmann distribution and the MPB model (Eq. 3) reduces to the PoissonBoltzmann model. ${ }^{56}$

Boundary conditions. - The potential at all anode/electrolyte interfaces was specified and equal to $\psi_{s}$. This potential was representative of actual experiments reported in the literature. ${ }^{21,47}$ The potential at the plane of symmetry between the anode and cathode was imposed to be zero. Symmetry boundary conditions were imposed on all other boundaries.

Constitutive relationships.-Table I summarizes the electrolyte material properties and associated potential $\psi_{s}$ used in the numerical simulations presented in this paper. The material properties were chosen so that they closely match the experimental conditions reported in the literature, ${ }^{21,47}$ subject to the assumptions of the MPB model. Two non-aqueous electrolytes were considered for the simulations, namely TEMA-BF 4 in propylene carbonate (PC) and $\mathrm{TEA}_{-} \mathrm{BF}_{4}$ in acetonitrile (AN). Both electrolytes are binary and symmetric with $z=1.47$

Moreover, the relative permittivity $\varepsilon_{r}$ significantly decreases at large electric fields $\left(E \geq 10^{7} \mathrm{~V} / \mathrm{m}\right)$ due to solvent polarization. ${ }^{57}$ Here, the Booth model was used to account for the field-dependent permittivity of an isotropic electrolyte given by ${ }^{43,44}$

$$
\varepsilon_{r}(E)=n^{2}+\left(\varepsilon_{r}(0)-n^{2}\right) \frac{3}{\beta E}\left(\operatorname{coth}(\beta E)-\frac{1}{\beta E}\right)
$$


where $E=|-\nabla \psi|$ is the norm of the electric field vector, $\varepsilon_{r}(0)$ is the relative permittivity of the solvent at zero electric field, $n$ is the index of refraction of the solvent. The coefficient $\beta$ was expressed as $^{57}$

$$
\beta=\frac{5 \mu}{2 k_{B} T}\left(n^{2}+2\right)
$$

where $\mu$ is the dipole moment of the solvent molecule. For AN, Eq. 5 predicts $\beta=30.15 \mathrm{~nm} / \mathrm{V}$ using the properties given in Table I. In addition, Wang et al. ${ }^{45}$ determined the value of $\beta$ for PC to be $13.14 \mathrm{~nm} / \mathrm{V}$ by least-square fitting the molecular dynamics simulation results reported in Ref. 58. In fact, results of molecular dynamics simulations for different electrolytes ${ }^{58,59}$ have verified that the Booth model accurately predicts the electrolyte permittivity for high electric fields up to $4 \mathrm{~V} / \mathrm{nm}$ typically encountered in EDLCs. ${ }^{58}$ Thus, the MPB model combined with the Booth model appears to be a more accurate and physically sound approach to simulate EDLCs under actual operating conditions.

The MPB model used assumes that both the cation and anion have the same effective ion diameter $a$. However, determining its value is not trivial, and in many cases it is considered to be a fitting parameter. ${ }^{18}$ The non-solvated diameters of the TEMA ${ }^{+}$cation and $\mathrm{BF}_{4}^{-}$anion were reported to be $0.68 \mathrm{~nm}^{39,47,60}$ and $0.44 \mathrm{~nm},{ }^{47}$ respectively. When the electrolyte concentration increases, the dissolved electrolyte ions are less solvated and their effective diameter decreases. ${ }^{46,61}$ Considering the fact that the solubility of TEMA- $-\mathrm{BF}_{4}$ in $\mathrm{PC}$ is about $2 \mathrm{~mol} / \mathrm{l}$ at room temperature, ${ }^{62}$ the effective ion diameter for the non-aqueous TEMA-BF 4 electrolyte was assumed to be slightly larger than the non-solvated diameter and equal to $0.7 \mathrm{~nm}$ at $c_{\infty}=1 \mathrm{~mol} / \mathrm{l}$. The non-solvated diameter of the $\mathrm{TEA}^{+}$cation was reported to be $0.686 \mathrm{~nm} .{ }^{63}$ Considering that the maximum solubility of $\mathrm{TEA}^{-\mathrm{BF}_{4}}$ in $\mathrm{AN}$ is about $1.5 \mathrm{~mol} / \mathrm{l}$ at room temperature, ${ }^{64}$ the effective ion diameter for the nonaqueous TEA-BF $\mathrm{B}_{4}$ electrolyte was assumed to be equal to $0.69 \mathrm{~nm}$ at $c_{\infty}=1 \mathrm{~mol} / \mathrm{l}$.

Data processing.-To assess the performance of the EDLCs simulated, the total charge stored at an electrode due to the diffuse layer capacitance, denoted by $Q$, was calculated by integrating the surface charge density over the electrode/electrolyte interface of surface area $A$, i.e., ${ }^{13}$

$$
Q=\int_{A} \varepsilon_{r} \varepsilon_{0}(\mathbf{n} \cdot \nabla \psi) d A
$$

where $\mathbf{n}$ is the outward unit normal vector at the electrode/electrolyte interface. In addition, the diffuse layer specific area capacitance of a single electrode denoted by $C_{s}^{D}$ (in $\mathrm{F} / \mathrm{m}^{2}$ ) is defined as

$$
C_{s}^{D}=\frac{Q}{\psi_{s} A}
$$

where $\psi_{s}$ is the potential drop across one half of the simulated EDLCs. Moreover, when the Stern layer specific area capacitance $C_{s}^{S t}$ is also accounted for, it is treated in series with the diffuse layer capacitance $C_{s}^{D}$ to give the total specific area capacitance, $C_{s}$ as

$$
\frac{1}{C_{s}}=\frac{1}{C_{s}^{D}}+\frac{1}{C_{s}^{S t}}
$$

Finally, the diffuse layer and total gravimetric capacitances $C_{g}^{D}$ and $C_{g}($ in $\mathrm{F} / \mathrm{g}$ ) are defined as

$$
C_{g}^{D}=C_{s}^{D} A_{s p} \quad \text { and } \quad C_{g}=C_{s} A_{s p}
$$

where the specific surface area denoted by $A_{s p}$ (expressed in $\mathrm{m}^{2} / \mathrm{g}$ ) is defined as the ratio of the surface area and the mass of the electrode. For the simulated geometry shown in Fig. 1, it simplifies to

\begin{abstract}
Table I. Properties of non-aqueous TEMA-BF 4 in propylene carbonate (PC), and non-aqueous TEA-BF 4 in acetonitrile ( $\mathrm{AN}$ ) used in the simulations (Refs. 45, 65-69).
\end{abstract}

\begin{tabular}{lcc} 
Property & $\begin{array}{c}\text { Non-aqueous } \\
\text { TEMA-BF }_{4} \text { in PC }\end{array}$ & $\begin{array}{c}\text { Non-aqueous } \\
\text { TEA-BF }_{4} \text { in AN }\end{array}$ \\
\hline $\begin{array}{l}\text { relative permittivity, } \varepsilon_{r}(0) \\
\text { valency, } z\end{array}$ & 64.4 (Ref. 65) & 35.97 (Ref. 67) \\
$\begin{array}{l}\text { dipole moment of } \\
\text { solvent molecule, } \mu\end{array}$ & 1 & 1 \\
$\beta$ (Eq. 5) & - & $1.308 \times 10^{-29} \mathrm{Cm}$ \\
& $13.14 \mathrm{~nm} / \mathrm{V}$ & $30.15 \mathrm{~nm} / \mathrm{V}$ \\
refraction index, $n$ & $($ Ref. 45$)$ & \\
effective ion diameter, $a$ & $1.42($ Ref. 66) & $1.34($ Ref. 68) \\
bulk molar concentration & $0.7 \mathrm{~nm}$ & $0.69 \mathrm{~nm}$ \\
of ions, $c_{\infty}$ & $1 \mathrm{M}$ & $1 \mathrm{M}$ \\
electrode potential, $\psi_{s}$ & $1.35 \mathrm{~V}$ & $1 \mathrm{~V}$
\end{tabular}

$$
A_{s p}=\frac{r+2 t \phi}{r t \rho_{e f f}}
$$

where $\rho_{\text {eff }}$ is the effective density of the electrode given by $\rho_{\text {eff }}=\rho_{c}(1-\phi)$ where $\phi$ is the electrode porosity and $\rho_{c}$ is the bulk density of graphite equal to $2.2 \mathrm{~g} / \mathrm{cm}^{3} .^{21}$

Method of solution.-The MPB model (Eq. 3) was implemented in the finite element package COMSOL 3.5a along with the associated boundary conditions and constitutive relationships. The equations were solved using the PARDISO solver. The simulations were run on a Dell Precision 690 workstation with two $2.66 \mathrm{GHz}$ QuadCore Intel Xeon CPUs and 40 GB of RAM. In order to validate the proper implementation of the governing equations, the numerical results were compared with those reported by Kilic et al. ${ }^{17}$ for a planar EDLC. Good agreement was found in all the cases considered.

Numerical convergence studies were performed to obtain the optimum mesh refinement for all the models considered. Tetrahedral quadratic Lagrange elements were used in all the meshes generated. Mesh refinement was required near the electrode surface where the potential drop was significant. Therefore, the meshing procedure consisted of specifying a maximum element size of $10 \mathrm{~nm}$ for the entire domain and another maximum element size of $0.3 \mathrm{~nm}$ for the electrode surface boundaries with a specified element growth rate of 1.05. The convergence criterion was chosen such that the maximum relative difference in the total charge stored, $Q$, was less than $1 \%$ when decreasing the maximum element size for the electrode surface boundaries by a factor of 0.8 . The degrees of freedom (DOF) for the meshes varied between 7000 and 200,000 depending on the pore radius.

\section{Results and Discussion}

The following subsections discuss the effects of the pore radius, field-dependent electrolyte permittivity, electrode porosity, effective ion diameter, and electrolyte properties on the diffuse layer capacitance of the idealized EDLC configuration described in the previous section. Finally, comparisons between predicted total gravimetric capacitance and experimental results reported in the literature ${ }^{21,47}$ are also presented. The bulk molar concentration of the non-aqueous electrolyte (PC or AN) in all simulations was assumed to be $c_{\infty}=1.0 \mathrm{~mol} / \mathrm{l}$. The surface potential $\psi_{s}$ was set at 1.35 and $1.0 \mathrm{~V}$ for electrolyte TEMA-BF 4 in PC and TEA-BF ${ }_{4}$ in $\mathrm{AN}$, respectively.

Effect of pore radius.-Figure 2 shows the variation of the predicted diffuse layer gravimetric capacitance $C_{g}^{D}$ as a function of the specific surface area $A_{s p}$ for TEMA-BF $\mathrm{B}_{4}$ in PC assuming constant relative permittivity $\varepsilon_{r}=\varepsilon_{r}(0)=64.4$. Note that the lines are intended only to guide the eyes. The diffuse layer gravimetric 
capacitance was found to increase almost linearly with specific surface area $A_{s p}$ up to $\sim 400 \mathrm{~m}^{2} / \mathrm{g}$ and then remained nearly constant around $100 \mathrm{~F} / \mathrm{g}$ for larger values of $A_{s p}$. This trend was also observed experimentally by Barbieri et al. ${ }^{2 \mathrm{P}}$ when measuring the capacitance of different carbon material EDLCs. The authors concluded that using extremely high surface area carbon electrodes for EDLCs may be "unprofitable." 21 They suggested that this limitation was caused by the space constriction for charge accommodation within the pore walls ${ }^{21}$ whose thickness decreases as the specific surface area increases. For a critical surface area, the pore wall thickness becomes similar to the screening length of the electric field. Then, the pore wall cannot accommodate the same amount of charge, resulting in the observed saturation of the gravimetric capacitance. ${ }^{21}$ However, the numerical results reported in Fig. 2 ignored the wall thickness and properties of the electrode material since only the electrolyte region of the EDLC was simulated. Therefore, the trend observed in Fig. 2 can be explained by the fact that, as the pores become smaller, the electrode surfaces surrounding the pores at potential $\psi_{s}$ get closer thereby reducing the electric field developing within the pores. This, in turn, reduces the charge stored at the electrode surface as defined by Eq. 6. This interpretation was also established by Wang et al. ${ }^{45}$ who computed the electric field near the surface in mesoporous electrodes consisting of closely packed spheres.

Effect of Field-dependent Permittivity.-Figure 2 also compares the diffuse layer gravimetric capacitance $C_{g}^{D}$ as a function of $A_{s p}$ for constant and field-dependent permittivity for TEMA-BF 4 in $\mathrm{PC}$ with $c_{\infty}=1 \mathrm{~mol} / \mathrm{l}, a=0.7 \mathrm{~nm}$, and $\phi=0.55$. The field-dependent permittivity $\varepsilon_{r}(E)$ was given by Eq. 4 with parameters summarized in Table I. ${ }^{65,66}$ Figure 2 shows that $C_{g}^{D}$ was systematically smaller when accounting for field-dependent permittivity compared with constant permittivity $\varepsilon_{r}=64.4$. This was attributed to the reduction in dielectric constant caused by the large electric field encountered near the electrode surface. Both cases showed the same general trend where $C_{g}^{D}$ increased almost linearly with $A_{s p}$ and then reached a plateau beyond a critical value of specific surface area. In addition, the slope of the linear part of the curve was smaller for the fielddependent permittivity compared with constant permittivity. Also, the plateau was reached for a larger specific surface area around $1200 \mathrm{~m}^{2} / \mathrm{g}$ instead of $400 \mathrm{~m}^{2} / \mathrm{g}$, as previously discussed. Note that Barbieri et al. ${ }^{21}$ observed, experimentally, a critical surface area around $1200 \mathrm{~m}^{2} / \mathrm{g}$ albeit for TEA-BF 4 in $\mathrm{AN}$.

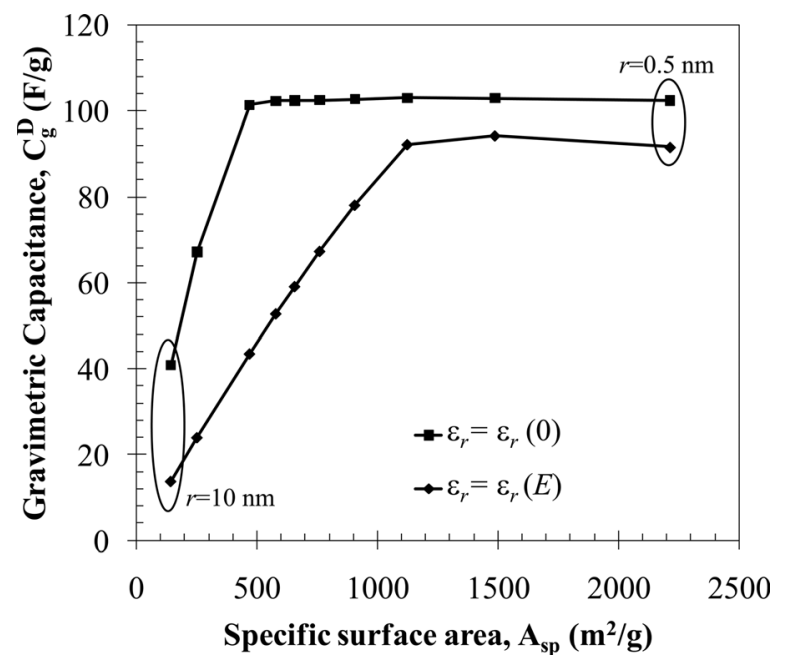

Figure 2. Effect of field-dependent permittivity on the diffuse layer gravimetric capacitance $C_{g}^{D}$ as a function of specific surface area $A_{s p}$ for TEMA$\mathrm{BF}_{4}$ in $\mathrm{PC}$ electrolyte. The relative permittivity was assumed to be either constant $\left[\varepsilon_{r}(0)=64.4\right]$ or field-dependent (Eq. 4). Here, $a=0.7 \mathrm{~nm}$, $\psi_{s}=1.35 \mathrm{~V}, c_{\infty}=1 \mathrm{~mol} / 1$, and $\phi=0.55$. The specific surface area was varied by changing the pore diameter $r$ from 0.5 to $10 \mathrm{~nm}$.
Effect of porosity.-To investigate the effect of porosity $\phi$ on the diffuse layer gravimetric capacitance $C_{g}^{D}$, the electrode porosity $\phi$ was set arbitrarily at $0.65,0.55$, and 0.35 , corresponding to geometric parameter $f=1.1,1.2$, and 1.5 , respectively. For all values of $\phi$, the radius of the cylindrical pores ranged from 0.5 to $10 \mathrm{~nm}$. The non-aqueous TEMA-BF 4 in PC electrolyte was considered using the MPB model assuming $a=0.7 \mathrm{~nm}$ and field-dependent permittivity $\varepsilon_{r}(E)$ given by Eq. 4 and parameters summarized in Table I.

Figure 3 a shows the predicted diffuse layer gravimetric capacitance $C_{g}^{D}$ as a function of specific surface area $A_{s p}$ for the three different values of $\phi$ considered. The trend was similar to those shown in Fig. 2 for all porosity values. It is evident that $C_{g}^{D}$ for small values of $A_{s p}$ was nearly the same regardless of porosity. In addition, its maximum value increased with increasing electrode porosity. The critical specific surface area beyond which $C_{g}^{D}$ reaches a plateau increased as porosity increased but corresponds to the same pore size $r=1 \mathrm{~nm}$ for all three porosities considered. This is due to the fact that, for a given pore radius $r, A_{s p}$ is linearly proportional to $\phi$ and the diffuse layer specific area capacitance $C_{s}^{D}=C_{g}^{D} / A_{s p}$ is the same regardless of porosity as confirmed by Fig. $3 \mathrm{~b}$.

Effect of ion size.-Here, three different values of effective ion diameter were considered, namely $a=0.5,0.7$, and $0.93 \mathrm{~nm}$. The upper limit for the effective ion diameter was based on the constraint that the packing parameter $v$ cannot exceed 1.0. ${ }^{19}$ For all simulations, the porosity was kept constant and equal to $\phi=0.55$. The non-aqueous $\mathrm{TEMA}^{-\mathrm{BF}_{4}}$ in PC electrolyte with field-dependent permittivity was considered.

Figure 4 shows the diffuse layer gravimetric capacitance $C_{g}^{D}$ versus specific surface area $A_{s p}$ for each value of the effective ion diameter. It indicates that $C_{g}^{D}$ increased significantly as the effective ion diameter decreased. This was particularly true for small pore radius and large specific surface area $A_{s p}$. It can be explained by the fact that as the effective ion diameter decreases, more ions can occupy the region near the electrode surface, thereby increasing the diffuse layer gravimetric capacitance. In fact, in the limiting case of $a=0$ $\mathrm{nm}$, the capacitance increased to unrealistic values as predicted by the PB model. ${ }^{17}$

Effect of electrolyte properties.-The electrolyte affects the performance of EDLCs not only through the effective ion diameter $a$ and the applied potential $\psi_{s}$ but also through the value of the parameters $\varepsilon_{r}(0)$ and $\beta$ in Eq. 4 . First, the effect of the zero field permittivity $\varepsilon_{r}(0)$ was investigated by simulating EDLCs with electrolyte featuring field-dependent permittivity with various $\varepsilon_{r}(0)$ while all other properties were those of TEMA-BF 4 in PC (Table I). In all cases, $a, c_{\infty}$, and $\phi$ were assumed to be $0.7 \mathrm{~nm}, 1 \mathrm{~mol} / \mathrm{l}$, and 0.55 , respectively.

Figure 5 plots the diffuse layer gravimetric capacitance $C_{g}^{D}$ versus specific surface area $A_{s p}$ for $\varepsilon_{r}(0)=40,64.4$, and 80 . All three curves show similar trends and values regardless of pore radius. In particular, the maximum value of $C_{g}^{D}$ obtained for $A_{s p}$ larger than $1400 \mathrm{~m}^{2} / \mathrm{g}$ was about $92 \mathrm{~F} / \mathrm{g}$ for all values of $\varepsilon_{r}(0)$ considered. Figure 5 also indicates that the slope of the linear part of the curve increased as $\varepsilon_{r}(0)$ increased from 40 to 80 . This behavior can be explained by the fact that for a given pore size in the linear regime, a larger value of $\varepsilon_{r}(0)$ and thus $\varepsilon_{r}(E)$ resulted in a larger value of $Q$, as suggested by Eq. 6 . Consequently, the critical value of specific surface area beyond which the capacitance reached a maximum decreases slightly with increasing $\varepsilon_{r}(0)$.

Moreover, Figure 6a plots the diffuse layer gravimetric capacitance $C_{g}^{D}$ as a function of $\varepsilon_{r}(0)$ between 10 and 150 for pore radius $r=1 \mathrm{~nm}$ corresponding to $\phi=0.55$ and $A_{s p}=1124 \mathrm{~m}^{2} / \mathrm{g}$. These configuration corresponds to the maximum capacitance shown in Figs. 2, 3 and 5. All other properties remained the same as those for Fig. 5. It shows that $C_{g}^{D}$ increased almost linearly from $60 \mathrm{~F} / \mathrm{g}$ to about $95 \mathrm{~F} / \mathrm{g}$ as $\varepsilon_{r}(0)$ varied from 10 to 70 . However, it remained nearly constant for $\varepsilon_{r}(0)$ larger than 70 . This indicates that there is 
(a)

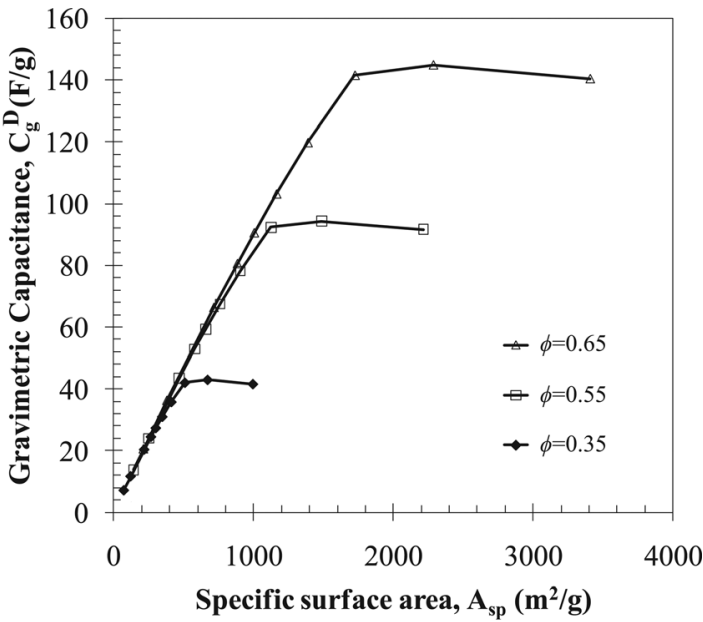

(b)

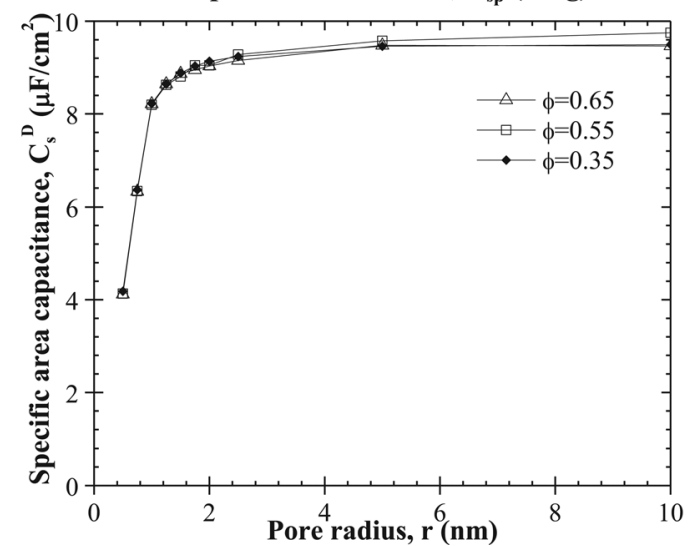

Figure 3. Effect of electrode porosity $\phi$ on the (a) diffuse layer gravimetric capacitance $C_{g}^{D}$ as a function of specific surface area $A_{s p}$ by varying pore radius $r$ from 10 to $0.5 \mathrm{~nm}$, and (b) diffuse layer specific area capacitance $C_{s}^{D}$ as a function of pore radius $r$ for TEMA-BF 4 in PC electrolyte. The porosity was taken as $\phi=0.35,0.55$, and 0.65 . Here, $a=0.7 \mathrm{~nm}, \psi_{s}=1.35 \mathrm{~V}$, $c_{\infty}=1 \mathrm{~mol} / \mathrm{l}$, and $\varepsilon_{r}=\varepsilon_{r}(E)$ given by Eq. 4 with parameters from Table I.

no benefit in terms of diffuse layer gravimetric capacitance from increasing $\varepsilon_{r}(0)$ beyond a certain threshold.

Similarly, the effect of $\beta$ on the diffuse layer gravimetric capacitance $C_{g}^{D}$ was investigated. Figure $6 \mathrm{~b}$ plots $C_{g}^{D}$ versus $\beta$ for pore radius $r=1 \mathrm{~nm}$ and $\varepsilon_{r}(0)=64.4$. It shows that $C_{g}^{D}$ decreased from $96 \mathrm{~F} / \mathrm{g}$ to about $60 \mathrm{~F} / \mathrm{g}$ as the value of $\beta$ increased from 10 to $100 \mathrm{~nm} / \mathrm{V}$. Thus, for a given value of $\varepsilon_{r}(0)$, it is desirable to find an electrolyte with the smallest value of $\beta$ in order to maximize the performance of EDLCs.

Predicting experimental data.-Wang et al. ${ }^{47}$ measured capacitances for EDLCs made from different samples of commercially available activated carbon covering a wide range of microporous and mesoporous morphology and surface area. They used TEMA$\mathrm{BF}_{4}$ in $\mathrm{PC}$ as the electrolyte with concentration $c_{\infty}=1 \mathrm{~mol} / \mathrm{l}$. Electrodes were prepared by mixing activated carbon powder with carbon black and a PTFE binder in an 80:10:10 mass ratio. The capacitance was determined using a two-electrode cell and the chargedischarge tests were carried out for a potential range between 0.1 and $2.7 \mathrm{~V}$. The specific surface area ranged from approximately 550 $\mathrm{m}^{2} / \mathrm{g}$ to about $2,050 \mathrm{~m}^{2} / \mathrm{g}$. Unfortunately, the electrode porosity was not reported.

Numerical simulations were performed for porosity of $\phi=0.55$ and the morphology described in Fig. 1 while the pore radius varied from 0.5 to $10 \mathrm{~nm}$ as previously discussed. The effective ion diameter for the TEMA-BF 4 in PC electrolyte was assumed to be $0.7 \mathrm{~nm}$ for bulk concentration $c_{\infty}=1 \mathrm{~mol} / \mathrm{l}$. As previously discussed, this corresponds to the ion diameter of non-solvated TEMA ${ }^{+}$(Refs. 39,

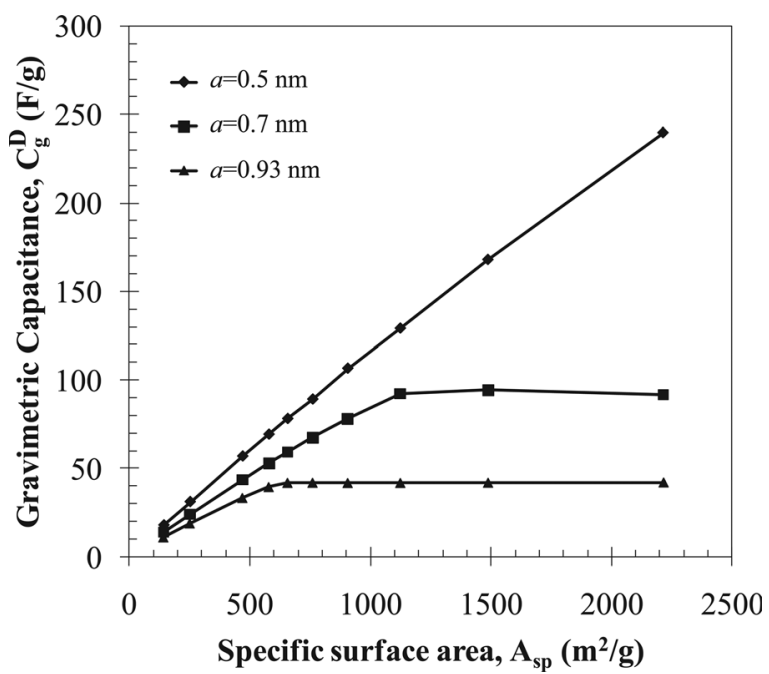

Figure 4. Effect of the effective ion diameter on the diffuse layer gravimetric capacitance $C_{g}^{D}$ as a function of specific surface area $A_{s p}$ by varying pore radius $r$ from 10 to $0.5 \mathrm{~nm}$ for TEMA-BF $\mathrm{BF}_{4}$ in $\mathrm{PC}$. The effective ion diameter was taken as $a=0.5,0.7$, or $0.93 \mathrm{~nm}$. Here, $\psi_{s}=1.35 \mathrm{~V}, c_{\infty}=1 \mathrm{~mol} / \mathrm{l}$, $\phi=0.55$, and field-dependent permittivity (Eq. 4).

47, and 60) and $\mathrm{TEA}^{+} .{ }^{63}$ The numerical simulations considered the field-dependent permittivity of the electrolyte. The Stern layer specific area capacitance $C_{s}^{S t}$ was assumed to be constant and considered in series with the diffuse layer specific area capacitance $C_{g}^{D}$ to achieve better agreement with the experimental data, as performed in the literature. ${ }^{18}$ Here, $C_{s}^{S t}$ was taken as $C_{s}^{S t}=10 \mu \mathrm{F} / \mathrm{cm}^{2}$. Note that this was the only parameter adjusted to achieve good agreement between numerical simulations and experimental data. Figure 7 compares the predicted total gravimetric capacitance $C_{g}$ as a function of specific surface area $A_{s p}$ with experimental results. ${ }^{47}$ Overall, the numerical simulations predicted similar trends and values for total gravimetric capacitance as those observed experimentally. ${ }^{47}$

Barbieri et al. ${ }^{21}$ also conducted experiments with EDLCs prepared using various commercially available carbon black samples as well as wood-based samples. The authors used non-aqueous TEA-

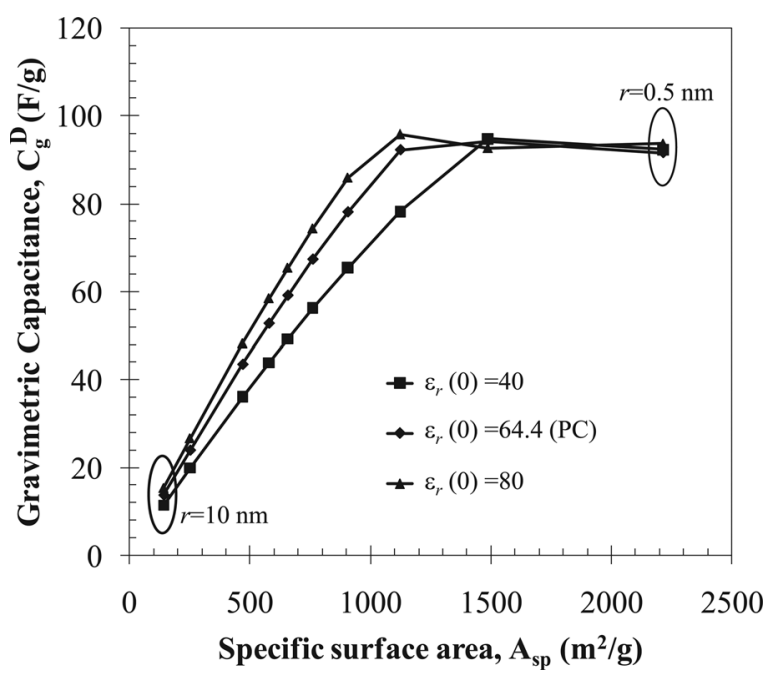

Figure 5. Effect of the relative permittivity $\varepsilon_{r}(0)$ on the diffuse layer gravimetric capacitance $C_{g}^{D}$ as a function of specific surface area $A_{s p}$. Here, $a=0.7 \mathrm{~nm}, \psi_{s}=1.35^{g} \mathrm{~V}, c_{\infty}=1 \mathrm{~mol} / \mathrm{l}, \phi=0.55$, and $\varepsilon_{r}=\varepsilon_{r}(E)$ given by Eq. 4 . The value of $\varepsilon_{r}(0)$ was $40,64.4$, and 80 while other parameters were those for TEMA-BF 4 in PC (Table I). 
(a)

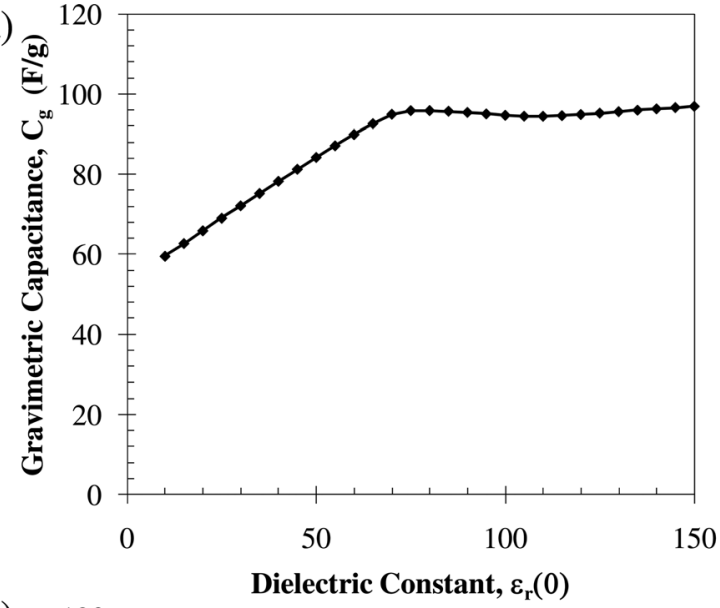

(b)

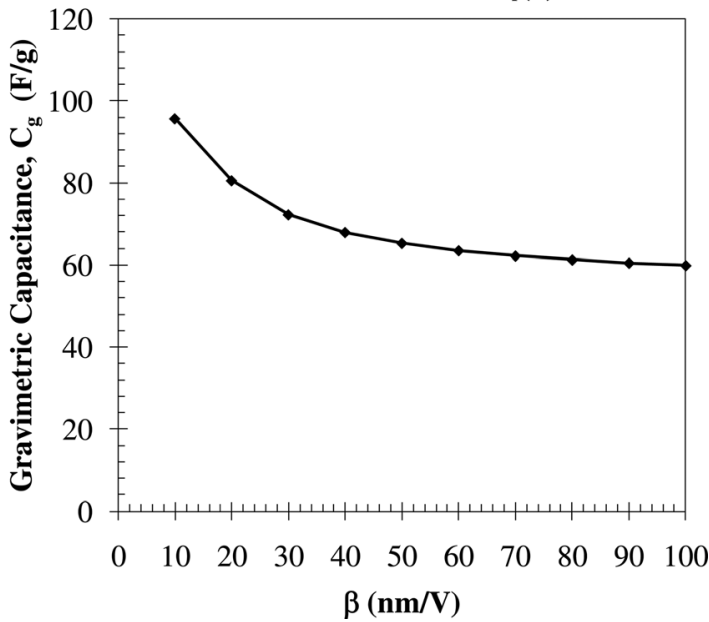

Figure 6. Effects of (a) the relative permittivity $\varepsilon_{r}(0)$, and (b) parameter $\beta$ on the diffuse layer gravimetric capacitance $C_{g}^{D}$ for $\mathrm{r}=1 \mathrm{~nm}$. Here, $a=0.7$ $\mathrm{nm}, \psi_{s}=1.35 \mathrm{~V}, c_{\infty}=1 \mathrm{~mol} / \mathrm{l}, \phi=0.55$, and $\varepsilon_{r}=\varepsilon_{r}(E)$ given by Eq. 4 with properties, other than $\varepsilon_{r}(0)$, of TEMA-BF ${ }_{4}$ in PC.

$\mathrm{BF}_{4}$ in $\mathrm{AN}$ electrolyte at $c_{\infty}=1 \mathrm{~mol} / \mathrm{l}$. A three-electrode cell was used to determine the capacitance of a single electrode by cyclic voltammetry with a potential window of -1 to $1 \mathrm{~V}$. The specific surface area ranged from about 130 to $1745 \mathrm{~m}^{2} / \mathrm{g}$. Here also, the electrode porosity was not reported and therefore the numerical simulations were performed for porosity of $\phi=0.55$ and assuming fielddependent permittivity $\varepsilon_{r}(E)$. The effective ion diameter for the TEA-BF ${ }_{4}$ in AN electrolyte was assumed to be $0.69 \mathrm{~nm}$ as previously discussed. Here also, $C_{s}^{S t}$ was taken as $10 \mu \mathrm{F} / \mathrm{cm}^{2}$. This value is lower than that computed by Wang et al ${ }^{45}\left(C_{s}^{S t}=20.3 \mu \mathrm{F} / \mathrm{cm}^{2}\right)$ based on the Helmholtz model for $1 \mathrm{~mol} / \mathrm{l} \mathrm{TEA}-\mathrm{BF}_{4}$ in PC with surface potential $\psi_{s}=0.75 \mathrm{~V}$.

Figure 8 shows the predicted total gravimetric capacitance $C_{g}$ as a function of the specific surface area $A_{s p}$ with that obtained experimentally. ${ }^{47}$ Here also, the numerical simulations predicted similar trends and values of $C_{g}$ as those obtained experimentally for the range of $A_{s p}$ considered. These results are satisfactory considering that the numerical simulations were performed for an idealized geometry (Fig. 1) and experimental data cover a wide range of porosity and pore size. They demonstrate that it is possible to predict the experimentally measured gravimetric capacitance of a wide range of electrode morphologies with a limited number of adjusted parameter, namely $C_{s}^{S t}$.

\section{Conclusion}

This study numerically solved the modified Poisson-Boltzmann model in EDLCs with mesoporous electrodes using the finite ele-

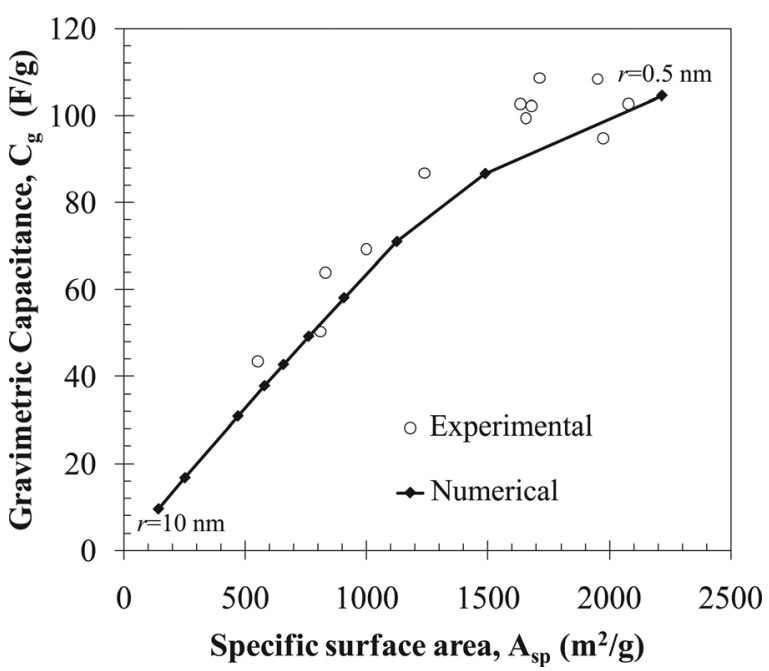

Figure 7. Comparison of experimental (Ref. 47) and numerically predicted total gravimetric capacitance $C_{g}$ as a function of specific surface area for TEMA-BF $F_{4}$ in PC electrolyte using field-dependent permittivity (Eq. 4) with $a=0.7 \mathrm{~nm}, c_{\infty}=1 \mathrm{~mol} / 1, \phi=0.55$, and $C_{s}^{S t}=10 \mu \mathrm{F} / \mathrm{cm}^{2}$.

ment method. Three-dimensional models were used to simulate EDLCs with mesoporous electrodes consisting of cylindrical pores. Parametric studies were performed to systematically investigate the effects of (i) pore radius, (ii) electrolyte field-dependent permittivity, (iii) porosity, (iv) effective ion diameter, and (v) electrolyte properties on the diffuse layer gravimetric capacitance of EDLCs. They established that reducing the ion effective diameter and the pore radius resulted in the strongest increase in diffuse layer gravimetric capacitance. However, the simulations showed that reducing the pore radius below a critical value did not give additional gains. They also stressed the need for reliable estimates of the effective ion size of the electrolyte to be used in the MPB model. In addition, increasing the electrode porosity significantly increased the diffuse layer gravimetric capacitance, particularly for smaller pore radius. To a lesser extent, accounting for the field-dependent permittivity, by using the Booth model for example, was found to reduce the predicted diffuse layer gravimetric capacitance. Then, a combination of small value of parameter $\beta(\leq 30 \mathrm{~nm} / \mathrm{V})$ and large $\varepsilon_{r}(0)(\geq 70)$ resulted in increased diffuse layer gravimetric capacitance. Finally,

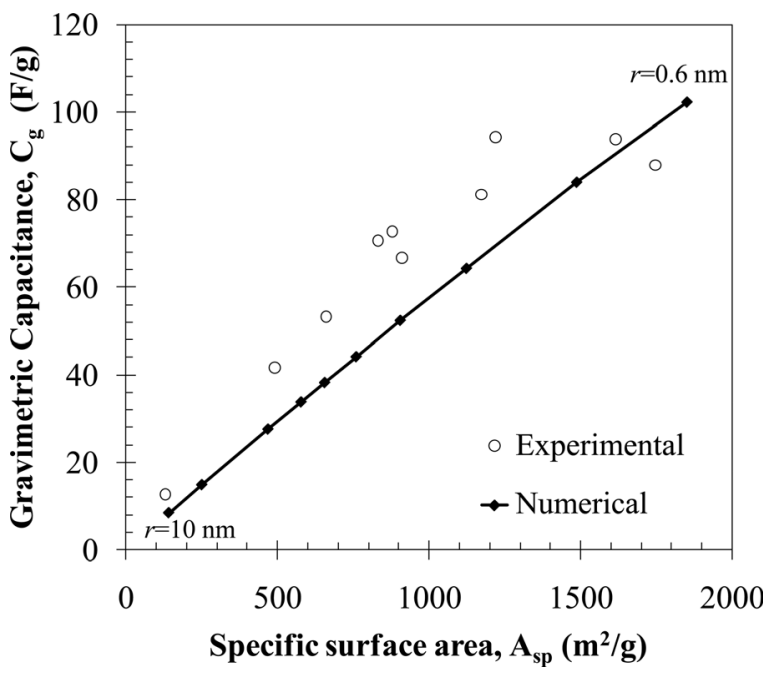

Figure 8. Comparison of experimental (Ref. 21) and numerically predicted total gravimetric capacitance $C_{g}$ as a function of specific surface area for TEA-BF 4 in $\mathrm{AN}$ electrolyte using field-dependent permittivity (Eq. 4) with $a=0.69 \mathrm{~nm}, c_{\infty}=1 \mathrm{~mol} / 1, \phi=0.55$, and $C_{s}^{S t}=10 \mu \mathrm{F} / \mathrm{cm}^{2}$. 
accounting for the contribution of the Stern layer to the total capacitance was essential in predicting experimental data for a wide range of electrode morphologies. Using the same constant Stern layer specific area capacitance of $C_{s}^{S t}=10 \mu \mathrm{F} / \mathrm{cm}^{2}$ in series with the diffuse layer capacitance gave good agreement with experimental data for both TEMA-BF 4 in $\mathrm{PC}^{47}$ and TEA-BF 4 in $\mathrm{AN} .^{21}$ The present approach could reduce the amount of extensive experimental testing required and guide the designers in increasing the performance of EDLCs.

\section{Acknowledgment}

This material is based upon work supported as part of the Molecularly Engineered Energy Materials, an Energy Frontier Research Center funded by the U.S. Department of Energy, Office of Science, Office of Basic Energy Sciences under Award Number DESC0001342.

\section{List of Symbols}

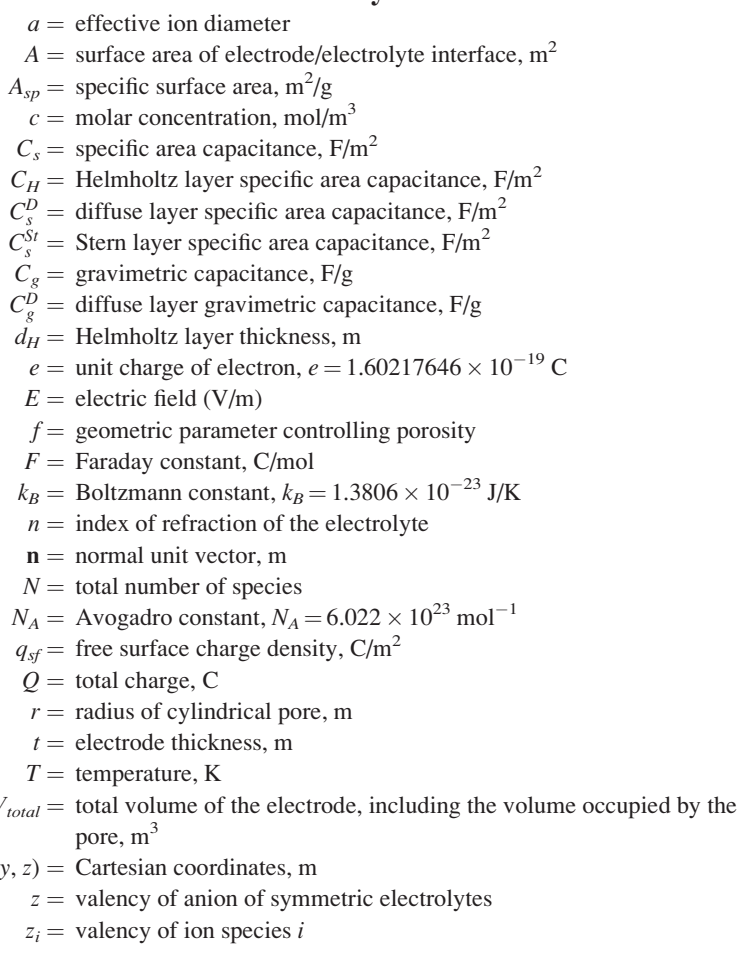

Greek Symbols

$\beta=$ coefficient in Booth model, $\mathrm{m} / \mathrm{V}$

$\varepsilon=$ permittivity, $\mathrm{F} / \mathrm{m}$

$\varepsilon_{0}=$ permittivity of free space, $\mathrm{F} / \mathrm{m}$

$\varepsilon_{r}(E)=$ relative permittivity at electric field $\mathrm{E}$

$\phi=$ porosity of electrode

$\psi=$ electric potential, $\mathrm{V}$

$\psi_{s}=$ specified potential on the anode surface, $\mathrm{V}$

$\psi_{\text {total }}=$ potential difference between the cathode and anode in the EDLC, $\mathrm{V}$

$v=2 \mathrm{a}^{3} \mathrm{~N}_{\mathrm{A}} \mathrm{c}_{\infty}$ packing parameter

$\mu=$ dipole moment of the solvent molecule, $\mathrm{C} \cdot \mathrm{m}$

$\rho_{\text {eff }}=$ effective density of the electrode, $\mathrm{g} / \mathrm{cm}^{3}$

$\rho_{c}=$ bulk density of graphite, $\rho_{c}=2.2 \mathrm{~g} / \mathrm{cm}^{3}$

Subscripts

$$
\begin{aligned}
i & =\text { refers to ion species } 1 \text { or } 2 \\
\max & =\text { refers to maximum possible value } \\
\infty & =\text { refers to bulk value }
\end{aligned}
$$

Superscripts

$D=$ refers to diffuse layer

$S t=$ refers to Stern layer

\section{References}

1. C. Vix-Guterl, E. Frackowiak, K. Jurewicz, M. Friebe, J. Parmentier, and F. Béguin, Carbon, 43, 1293 (2005)

2. E. Frackowiak, Carbon, 39, 937 (2001).

3. E. Frackowiak, Phys. Chem. Chem. Phys., 9, 1774 (2007).

4. L. L. Zhang and X. S. Zhao, Chem. Soc. Rev., 38, 2520 (2009).

5. US Department of Energy, Basic Research Needs for Electrical Energy Storage, http://www.osti.gov/accomplishments/documents/fullText/ACC0330.pdf, last accessed: July 25, 2007.

6. R. Kotz and M. Carlen, Electrochim. Acta, 45, 2483 (2000).

7. Y. Zhang, H. Feng, X. Wu, L. Wang, A. Zhang, T. Xia, H. Dong, X. Li, and L. Zhang, Int. J. Hydrogen Energy, 34, 4889 (2009).

8. R. J. Hunter, Foundations of Colloid Science, Vol. 1, Clarendon, Oxford (1987).

9. C. M. Brett and A. M. O. Brett, Electrochemistry Principles, Methods and Applications, Oxford University, Oxford (1993).

10. B. E. Conway, Electrochemical Supercapacitors: Scientific Fundamentals and Technological Applications, Kluwer Academic/Plenum Publishers, New York (1999).

11. G. Gouy, J. Phys. Theor. Appl., 9, 457 (1910).

12. D. L. Chapman, Philos. Mag., Ser. 6, 25, 475 (1913).

13. J. H. Masliyah and S. Bhattacharjee, Electrokinetic and Colloid Transport Phenomena, John Wiley \& Sons, Hoboken, NJ (2006).

14. O. Stern, Z. Elektrochem. Angew. Phys. Chem., 30, 508 (1924).

15. D. C. Grahame, Chem. Rev., 41, 441 (1947).

16. A. J. Bard and L. R. Faulkner, Electrochemical Methods: Fundamentals and Applications, Wiley and Sons, New York (2001).

17. M. S. Kilic, M. Z. Bazant, and A. Ajdari, Phys. Rev. E, 75, 1 (2007).

18. M. Z. Bazant, M. S. Kilic, B. D. Storey, and A. Ajdari, Adv. Colloid Interface Sci., 152, 48 (2009).

19. P. M. Biesheuvel and M. Van Soestbergen, J. Colloid Interface Sci., 316, 490 (2007).

20. H. Shi, Electrochim. Acta, 41, 1633 (1996).

21. O. Barbieri, M. Hahn, A. Herzog, and R. Kotz, Carbon, 43, 1303 (2005).

22. V. V. N. Obreja, Physica E (Amsterdam), 40, 2596 (2008).

23. D. N. Futaba, K. Hata, T. Yamada, T. Hiraoka, Y. Hayamizu, Y. Kakudate, O. Tanaike, H. Hatori, M. Yumura, and S. Iijima, Nature Mater., 5, 987 (2006).

24. C. Yu, C. Masarapu, J. Rong, B. Wei, and H. Jiang, Adv. Mater., 21, 4793 (2009).

25. Y. Honda, T. Ono, M. Takeshige, N. Morihara, H. Shiozaki, T. Kitamura, K. Yoshikawa, M. Morita, M. Yamagata, and M. Ishikawa, Electrochem. Solid-State Lett., 12, 45 (2009).

26. M. Sevilla, S. Alvarez, T. A. Centeno, A. B. Fuertes, and F. Stoeckli, Electrochim. Acta, 52, 3207 (2007).

27. J. Li, X. Wang, Q. Huang, S. Gamboa, and P. J. Sebastian, J. Power Sources, 158, 784 (2006).

28. T. Bordjiba, M. Mohamedi, and L. H. Dao, Adv. Mater., 20, 815 (2008).

29. Y.-S. Hu, P. Adelhelm, B. M. Smarsly, S. Hore, M. Antonietti, and J. Maier, Adv. Funct. Mater., 17, 1873 (2007).

30. S. S. Mao and X. Chen, Int. J. Energy Res., 31, 619 (2007).

31. Y. Lei, W. Cai, and G. Wilde, Prog. Mater. Sci., 52, 465 (2007).

32. J. Liu, G. Cao, Z. Yang, D. Wang, D. Dubois, X. Zhou, G. L. Graff, L. R. Pederson, and J.-G. Zhang, ChemSusChem, 1, 676 (2008).

33. F. Cheng, Z. Tao, J. Liang, and J. Chen, Chem. Mater., 20, 667 (2008).

34. A. Stein, Z. Wang, and M. A. Fierke, Adv. Mater., 21, 265 (2009).

35. M. Itagaki, New Carbon Mater., 24, 193 (2009).

36. J. Huang, B. G. Sumpter, and V. Meunier, Angew. Chem. Int. Ed., 47, 520 (2008).

37. J. Huang, B. G. Sumpter, and V. Meunier, Chem.-Eur. J., 14, 6614 (2008).

38. J. Huang, B. G. Sumpter, V. Meunier, G. Yushin, C. Portet, and Y. Gogotsi, J. Mater. Res., 25, 1525 (2010).

39. J. Chmiola, G. Yushin, Y. Gogotsi, C. Portet, P. Simon, and P. L. Taberna, Science, 313, 1760 (2006)

40. M. C. Henstridge, E. J. F. Dickinson, and R. G. Compton, Chem. Phys. Lett., 485, 167 (2010).

41. J. Huang, R. Qiao, B. G. Sumpter, and V. Meunier, J. Mater. Res., 25, 1469 (2010).

42. H. Wang and L. Pilon, J. Phys. Chem. C. (in press, 2011)

43. F. Booth, J. Chem. Phys., 19, 391 (1951).

44. F. Booth, J. Chem. Phys., 23, 453 (1955).

45. H. Wang, J. Varghese, and L. Pilon, Electrochem. Acta, 56, 6189 (2011).

46. H.-J. Liu, W.-J. Cui, L.-H. Jin, C.-X. Wang, and Y.-Y. Xia, J. Mater. Chem., 19, 3661 (2009).

47. L.-H. Wang, M. Toyoda, and M. Inagaki, New Carbon Mater., 23, 111 (2008).

48. J. N. Israelachvili, Intermolecular and Surface Forces, 2nd ed., Academic, London (1992).

49. L. Song, D. Feng, H. Lee, C. Wang, Q. Wu, D. Zhao, and B. D. Vogt, J. Phys. Chem. C, 114, 9618 (2010).

50. G. Yushin, A. Nikitin, and Y. Gogotsi, Nanomaterials Handbook, CRC, Boca Raton, FL (2006)

51. R. Qiao and N. R. Aluru, J. Chem. Phys., 118, 4692 (2003).

52. H. Daiguji, Chem. Soc. Rev., 39, 901 (2010).

53. J. C. T. Eijkel and A. van den Berg, Chem. Soc. Rev., 39, 957 (2010).

54. W. Sparreboom, A. van den Berg, and J. C. T. Eijkel, New J. Phys., 12, 1 (2010).

55. J. G. Ibarra-Armenta, A. Martin-Molina, and M. Quesada-Perez, Phys. Chem. Chem. Phys., 11, 309 (2009).

56. J. Lyklema, Fundamentals of Interface and Colloid Science, Volume I: Fundamentals, Academic, San Diego, CA (2001). 
57. V. N. Paunov, R. I. Dimova, P. A. Kralchevsky, G. Broze, and A. Mehreteab, J. Colloid Interface Sci., 182, 239 (1996).

58. L. Yang, B. H. Fishbine, A. Migliori, and L. R. Pratt, J. Chem. Phys., 132, 1 (2010).

59. R. Fulton, J. Chem. Phys., 130, 1 (2009).

60. M. Ue, K. Ida, and S. Mori, J. Electrochem. Soc., 141, 3336 (1994).

61. B. E. Conway, Annu. Rev. Phys. Chem., 17, 481 (1966).

62. T. Nishida, Y. Tashiro, and M. Yamamoto, J. Fluorine Chem., 120, 135 (2003).

63. M. Inagaki, H. Konno, and O. Tanaike, J. Power Sources, 195, 7880 (2010).

64. T. Devarajan, S. Higashiya, C. Dangler, M. Rane-Fondacaro, J. Snyder, and P. Haldar, Electrochem. Commun., 11, 680 (2009).
65. G. J. Janz and R. P. T. Tomkins, Nonaqueous Electrolytes Handbook, Vol. 1, Academic, New York (1972).

66. A. Pal, G. Dass, and A. Kumar, J. Chem. Eng. Data, 43, 738 (1998).

67. C. Wohlfarth, Dielectric Constant of Acetonitrile, Springer Materials-The Landolt-Börnstein Database, Vol. 17, Springer-Verlag, Berlin, Germany (2008).

68. C. Wohlfarth, Refractive Index of Acetonitrile, Springer MaterialsThe Landolt-Börnstein Database, Vol. 47, Springer-Verlag, Berlin, Germany (2008).

69. J. A. Dean, Lange's Handbook of Chemistry, McGraw-Hill, New York (1972) 